Relaciones de género en la escuela: entre la desnaturalización de los "micromachismos" y la reproducción de perspectivas binarias Analía Inés Meo

Lucía Elena Cavalo

DOI: https://doi.org/10.24215/16696581e188

\title{
Relaciones de género en la escuela: entre la desnaturalización de los "micromachismos" y la reproducción de perspectivas binarias
}

Gender relations in school: between unpacking "micromachisms" and the reproduction of binary perspectives

Analía Inés Meo analia.meo@gmail.com

https://orcid.org/0000-0003-3290-6459

Instituto de Investigaciones Gino Germani; Facultad de Ciencias Sociales; Universidad de Buenos Aires/ Consejo Nacional de Investigaciones Científicas y Técnicas

Lucía Elena Cavalo lucia.cavalo@hotmail.com

https://orcid.org/0000-0001-5253-1215

Facultad de Ciencias Sociales, Universidad de Buenos Aires/ Facultad de Ciencias Empresariales y Sociales 
Analía Inés Meo, Lucía Elena Cavalo Relaciones de género en la escuela: entre la desnaturalización de los “micromachismos” y la reproducción de perspectivas binarias

\section{Resumen}

Este artículo examina las características, límites y potencialidades de una "actividad" dedicada a problematizar las relaciones tradicionales entre varones y mujeres, llevada a cabo en una escuela secundaria universitaria técnica de la Ciudad de Buenos Aires. La misma se inscribe en una serie de iniciativas inter e intra institucionales -y extracurriculares- sobre "violencia hacia las mujeres" que, desde 2016, son impulsadas, promovidas y acompañadas por autoridades y docentes de esta escuela.

El análisis de esta "actividad" nos permite reconocer interrogantes y posibles caminos para continuar desentrañando las maneras en que cotidianamente la escuela produce y reproduce una variedad de prácticas y discursos que, por su iteración, participan activamente en la generización de sus integrantes. Asimismo, nos brinda una primera aproximación a discursos sociales que disputan los sentidos acerca de lo considerado deseable y apropiado para cada uno de los géneros, así como para las relaciones éstos entablan entre sí.

Palabras clave: Género; intervención pedagógica; heteronormatividad; machismo; escuela secundaria.

\section{Abstract}

This article portrays one pedagogic intervention aiming at problematizing "machismo" and traditional gender relations carried out in a technical secondary school in the City of Buenos Aires. This imitative is part and parcel of a wide range of inter and intra institutional activities addressing "violence against women", which have been run and promoted by the school authorities and different teachers since 2016. This article reveals the ways in which this pedagogic intervention contributes to the configuration of heteronormative approaches to gender and sexualities. Furthermore, it evidences how difficult for adults is to engage with young people's resistances and challenges to a binary approach to gender and violence.

Keywords: Gender; pedagogic intervention; heteronormativity; machismo; secondary school. 
Analía Inés Meo, Lucía Elena Cavalo Relaciones de género en la escuela: entre la desnaturalización de los “micromachismos” y la reproducción de perspectivas binarias

En nuestro país, desde la década de 1990, investigadores/as de distintas disciplinas se han dedicado a analizar los modos en que las escuelas participan de la (re)producción de normatividades sexo-genéricas con profundos efectos en las subjetividades de quienes las habitan cotidianamente (Goldstein y Glejzer, 2008; Morgade, 2002; Sharagrodsky, 2016; Ramos, 2009; Wainerman y Chami, 2014). Este artículo dialoga con las indagaciones locales sobre género y educación, a partir del análisis de los límites y potencialidades de una "actividad" escolar destinada a problematizar las relaciones tradicionales de género (1). La misma se inscribe en un amplio abanico de intervenciones pedagógicas sobre género realizadas en una escuela secundaria universitaria técnica de la Ciudad Autónoma de Buenos Aires, que responden a intereses y preocupaciones de sus integrantes (2). En este sentido, las autoridades de esta escuela han asumido un fuerte compromiso con la visibilización y problematización de la "violencia hacia las mujeres" promoviendo y acompañando -desde 2015- diversas iniciativas intra e interinstitucionales destinadas a problematizar -junto a estudiantes de distintos niveles-, aspectos de las relaciones tradicionales entre varones y mujeres con foco en distintas manifestaciones de violencia contra estas últimas.

En esta presentación se analiza una de estas "actividades" extracurriculares organizada en motivo de la tercera marcha conocida como "Ni Una Menos. Según la "profe coordinadora" que la ideó, su dinámica giró en torno a la identificación, por parte de alumnos/as pertenecientes a dos divisiones, de "micromachismos" en "spots publicitarios". En primer lugar, la elección de esta actividad se basa en que esta ofrece una oportunidad para reconocer maneras de (re)presentar las relaciones de género consideradas legítimas en la escuela (al menos por algunos adultos de la misma). Esto, permite empezar a mapear maneras en que la escuela busca generizar -y generiza- a sus miembros jóvenes y adultos, al inculcar visiones, valores y sentidos "acerca de lo que está bien y de lo que está mal, de lo permitido y de lo prohibido [en materia de géneros y sexualidades]" (Alonso, Herczeg y Zurbriggen, 2009: 216). En otras palabras, las formas en que la escuela nomina a las personas, lo que hacen y cómo deben (o no) relacionarse entre ellas participa activamente de los complejos procesos de producción de masculinidades y femeneidades; consolidando o desafiando -de maneras sutiles e inconscientes gracias a la iteración cotidiana de prácticas y discursos- el sistema de sexogénero heteronormativo dominante. En segundo lugar, la elección de esta "actividad" reside en que su devenir posibilitó reconocer que los/as jóvenes ofrecían visiones disonantes respecto de las de los/as adultos/as. Durante su transcurso, los/as estudiantes tensionaron los términos con los que los/as adultos buscaban encuadrar las relaciones entre varones y mujeres, intervenciones que estos últimos buscaron encauzar en términos dicotómicos -"machista" y "no machista"-.

Question, Vol. 1, N. ${ }^{\circ} 63$, julio-septiembre 2019. ISSN 1669-6581

Instituto de Investigaciones en Comunicación | Facultad de Periodismo y Comunicación Social | Universidad Nacional de La Plata La Plata | Buenos Aires | Argentina

Página 3 de 22 
Analía Inés Meo, Lucía Elena Cavalo Relaciones de género en la escuela: entre la desnaturalización de los “micromachismos” y la reproducción de perspectivas binarias

A continuación, se presenta la escuela de este estudio y la centralidad que en esta ha tenido la "lucha contra la violencia de género". En las secciones subsiguientes se describe y analiza la "actividad" sobre "micromachismos" ya mencionada, a partir de indagar los discursos sociales sobre los géneros que prevalecieron en los intercambios que se suscitaron entre jóvenes y adultos luego de la visualización de los "spots".

\section{La "lucha contra la violencia de género" como prioridad institucional}

La escuela Forjando Futuros fue creada en 2014 por una prestigiosa universidad pública, en el marco del Proyecto de creación de Nuevas Escuelas Secundarias con Universidades Nacionales lanzado por el Ministerio de Educación de la Nación. Se trata de una institución con modalidad técnica que busca "incluir" a jóvenes en "situación de vulnerabilidad", en un intento de revertir el carácter históricamente expulsivo tanto de esta modalidad como de la educación secundaria universitaria. A diferencia de otras escuelas similares dependientes de la misma universidad, ésta adopta una política no selectiva: la mayoría de las vacantes son asignadas por sorteo. En diciembre de 2017, esta escuela contaba con aproximadamente 220 alumnos distribuidos en siete divisiones y, aunque solo un tercio de su alumnado estaba conformado por mujeres, éstas tenían un peso relativo mayor al promedio total de las escuelas técnicas en la Ciudad. Esta escuela se encuentra en una de las comunas que registra peores indicadores sociales y educativos de la CABA (GCBA 2015). Por ejemplo, en relación a éstos últimos, esta comuna en 2014 tenía las tasas de sobre edad y repitencia en primaria (14,6\% y 2,3\% respectivamente) y secundaria más altas de la Ciudad (42,6\% de alumnos sobre edad y $11,8 \%$ de repetidores).

Durante nuestro trabajo de campo registramos cómo la escuela se iba construyendo material y simbólicamente. Sus miembros la definían con entusiasmo como una escuela "experimental", "nueva" y "fundacional", en la que "estaba todo por hacerse". Esta representación, los y las habilitaba a movilizar "expectativas", "proyectos" y "desafíos profesionales" en su producción cotidiana. Asimismo, manifestaban estar motivados por los niveles relativamente altos de autonomía con la que contaban para definir qué enseñar, cómo enseñar y cómo evaluar en pos de configurar una escuela "inclusiva" con "calidad educativa". Esta coyuntura institucional los habilitaba a proponer actividades fuera y dentro del horario escolar, movilizando sus propios intereses y saberes. 
Analía Inés Meo, Lucía Elena Cavalo Relaciones de género en la escuela: entre la desnaturalización de los “micromachismos” y la reproducción de perspectivas binarias

Una de las prioridades de esta escuela desde su fundación ha sido la explicita participación y promoción de iniciativas orientadas a "erradicar la violencia contra las mujeres"; un compromiso expresado por el rector y el vice-rector en reiteradas ocasiones. Esta preocupación -inscripta en el contexto-socio histórico de creciente presencia pública y legitimidad del movimiento para denunciar la violencia contra las mujeres y su manifestación más cruenta, el femicidio-, se materializó, por ejemplo, en acciones inter-institucionales en el territorio. Entre estas iniciativas se destacan: 1.la organización de una "caminata" "Este barrio dice 'Ni una Menos"' en la que participaron decenas de docentes y alumnos/as, e integrantes de agencias estatales y no estatales, en 2017 y en 2018; y 2. la organización de un "curso de formación" de "agentes de género" que tuvo lugar en la escuela, a la que asistieron miembros de distintas escuelas.

Otras acciones de este tipo fueron organizadas "desde abajo" y, aunque no eran parte del currículo escolar formal, contaron con el aval de directivos. Si bien estas actividades no fueron muy frecuentes, se fueron consolidando como un espacio legitimado institucionalmente donde abordar junto a algunos/as grupos de estudiantes distintas temáticas de género. Durante nuestro trabajo de campo, documentamos la realización de "talleres sobre género" optativos en 2016; la autorización a varios/as "profes coordinadores" para realizar diferentes "actividades" vinculadas a temáticas de género (la del 8 de marzo, "Día de la mujer trabajadora"; las realizadas durante la semana de la movilización de $\mathrm{Ni}$ Una Menos), y el reconocimiento del derecho de las trabajadoras de la escuela "a hacer paro de mujeres".

La mayoría de estas iniciativas fueron organizadas y llevadas adelante por "profes coordinadores", un cargo considerado novedoso en esta escuela que tiene por principal función liderar espacios de "acompañamiento pedagógico" y, en menor medida, realizar actividades de enseñanza frente a cursos (por ejemplo, coordinando actividades extra-curriculares de distinto tipo con los/as alumnos/as).

\section{La "actividad" por el "Ni una menos"}

La mañana del 2 de Junio de 2017, Leila (3) iba a trabajar con chicos y chicas de segundo año sobre unos "spots publicitarios (...) por el día de Ni una Menos", movilización que se llevaría a cabo al día siguiente. Ella eligió los materiales con los que trabajar en la "actividad", la cual tuvo una duración aproximada de una hora. Parte de ese tiempo fue cedido por un docente de "técnica" y otra parte fue tomada del horario de almuerzo, previo acuerdo entre uno de los asesores pedagógicos y varios profesores del área técnica. En tanto los "profes coordinadores" 
Analía Inés Meo, Lucía Elena Cavalo Relaciones de género en la escuela: entre la desnaturalización de los “micromachismos” y la reproducción de perspectivas binarias

y las autoridades entendían que ese tipo de "actividades" era parte de la "formación integral" de los/as estudiantes, la asistencia a estos espacios extracurriculares era obligatoria.

Al inicio de la actividad, la docente invitó a los estudiantes a clasificar (y con ello a valorar) lo que verían en los "spots" en binomios o pares dicotómicos: "machista"/"no machista", "sexista"/"no sexista". De esta manera, esta docente demarcó los objetivos de la actividad y las categorías que esperaba que los/as estudiantes utilizaran para interrogar los materiales visuales que se les mostraría. Leila presentó los objetivos de la actividad en los siguientes términos: "La idea es ver que les pareció. ¿Les pareció machista? ¿No? ¿Por qué? (...) Después en grupos que elijan una, la que más les gustó, la que más les copa para modificar". Durante la "actividad" se proyectaron un total de seis publicidades. Cada una de ellas se sucedió de una discusión iniciada y moderada por algún adulto. En general era la docente quien formulaba las preguntas o emitía los primeros comentarios. También Ricardo, un profesor de "fabricación" -quien no participó en la organización de la "actividad" pero sí de su desarrollo- hacía observaciones y ampliaba comentarios hechos por la docente. Fueron contadas las intervenciones de José, el otro profesor coordinador presente, y de Marga, una asesora pedagógica que ingresó por unos breves minutos al aula. Al finalizar cada "spot", luego de los primeros comentarios de los adultos, unos pocos varones y aún menos mujeres participaron con preguntas, cuestionamientos y/o haciendo bromas y referencias a sus propias vivencias y saberes en torno a las relaciones de género.

A continuación, presentaremos el análisis de los intercambios que se produjeron entre estudiantes y adultos en torno a los "spots" visualizados durante la "actividad", para luego adentrarnos en las conversaciones que tuvieron lugar hacia el cierre de la misma, cuando los/as jóvenes fueron invitados/as a elaborar carteles con frases alusivas a lo trabajado.

Desnaturalizando jerarquías entre varones y mujeres: entre la binarización y los puntos de fuga

Al comenzar la actividad, la docente hizo referencia a la movilización del "Ni Una Menos" que se llevaría a cabo al día siguiente como el evento en el que se enmarcaría esta propuesta. La mención a esta fecha sirvió como un puntapié para problematizar manifestaciones sutiles y cotidianas de "violencia de género", a las que Leila refirió como "micromachismos". En sus palabras, el foco de la actividad estuvo puesto en: 
Analía Inés Meo, Lucía Elena Cavalo Relaciones de género en la escuela: entre la desnaturalización de los “micromachismos” y la reproducción de perspectivas binarias

\begin{abstract}
"esas cosas que por ahí pasan desapercibidas (...) porque pareciera que está todo bien, que no nos dice nada raro, pero en realidad está reproduciendo estereotipos sociales de lo que el hombre debe ser y de lo que la mujer deber ser" (Leila sobre el "spot A")
\end{abstract}

En línea con este propósito, los anuncios publicitarios seleccionados pusieron en escena y permitieron cuestionar las jerarquías que estructuran las relaciones entre los géneros así como distintos tipos de violencia contra las mujeres. Estas últimas, hacían referencia, pero también rebasaban, la denuncia contra la "violencia física" ejercida por varones hacia mujeres que enarboló el movimiento Ni Una Menos en sus inicios. En otras palabras, la puesta en escena de esta "actividad" contribuyó a ingresar situaciones cotidianas que raramente son problematizadas como formas de violencia cuando se hace referencia al "Ni Una Menos".

Durante los intercambios, las palabras "machista", "sexista" y "micromachismo" organizaron la selección de los materiales, estructuraron los diálogos y condensaron los núcleos de sentido que se quisieron hacer circular y poner en juego. Los primeros dos términos ("machista" y "sexista"), fueron empleados por adultos para hacer referencia a relaciones no deseables entre "hombres" y "mujeres"; entendidas como aquellas en las que la mujer ocupa un lugar subordinado respecto al varón. En concordancia, el término "micromachismo" fue utilizado para problematizar y desnaturalizar algunas expresiones de violencia de género que, aunque más sutiles que las primeras, se encontraban presentes en las publicidades.

A continuación, examinaremos distintas aristas de las relaciones tradicionales entre varones y mujeres que fueron tematizadas por los adultos durante la "actividad", así como sus puntos de contacto y de fuga respecto a las interpretaciones de los y las estudiantes.

\title{
La división sexual del trabajo en jaque
}

De los seis anuncios visualizados, los spots A, B y C (ver abajo recuadros con descripciones) fueron utilizados como disparadores para problematizar lo que interpretamos como la tradicional división sexual del trabajo en la familia. En este sentido, las intervenciones de los/a adultos/a estuvieron dirigidas a cuestionar que las mujeres debieran ser las principales -sino únicas- responsables del trabajo reproductivo no remunerado. Para cuestionar estos aspectos, 
Analía Inés Meo, Lucía Elena Cavalo Relaciones de género en la escuela: entre la desnaturalización de los “micromachismos” y la reproducción de perspectivas binarias

se presentaron dos spots que publicitaban diferentes marcas de detergente (spot $B$ y $C$ ), un producto que según ella se encuentra "encasillado (...) con la casa, con la familia, con la mujer".

A continuación, se presenta una descripción de los anuncios elegidos por "la coordi" para problematizar esta temática:

\section{SPOT A}

La publicidad se inicia con la imagen de una mujer recostada sola en una cama matrimonial, con una computadora en su falda y una tarjeta de crédito en sus manos, denotando que se encuentra realizando una compra on-line. En otra habitación, que parece estar en la misma casa, un hombre cuida de manera poco exitosa a varios niños pequeños. Éstos corren, gritan, desordenan el cuarto, mientras el hombre permanece sentado, inmóvil. Casi sin poder reaccionar, superado por la situación, el hombre toma un Handy para comunicarse con la mujer y le pide disculpas a "águila madre" por "haber vuelto a las dos mil trescientas horas de trabajar toda la semana". Finalmente, la mujer abre la puerta de la habitación de los niños y al verlos durmiendo, se comunica por handy con el hombre diciéndole: "águila padre, la operación ha sido un éxito. Puede volver al nido".

\section{SPOT B}

Consiste en una sucesión de imágenes que muestran a un hombre adulto realizando distintas tareas de cuidado de una niña pequeña. La lleva al colegio, le prepara las comidas, la acuesta por la noche. En ningún momento se muestra a una mujer. Finaliza con el logo de una marca de detergente.

\section{SPOT C}

Comienza con un grupo de jóvenes (dos varones y una mujer) que lavan platos en distintas situaciones que, a juzgar por los diversos paisajes de fondo y su vestimenta de mochileros, denotaría que están realizando un viaje. Una voz en off, en nombre de la marca de detergente, invita a sumarse al desafío de recorrer Latinoamérica con una sola botella de este producto.

Question, Vol. 1, N. ${ }^{\circ}$ 63, julio-septiembre 2019. ISSN 1669-6581

Instituto de Investigaciones en Comunicación | Facultad de Periodismo y Comunicación Social | Universidad Nacional de La Plata 
La "coordi" (así se llama informalmente a los/as profesores coordinadores) afirmó que el "spot A" era "machista" porque reproducía "estereotipos sociales" de género, en tanto prescribían que "el hombre tiene que trabajar, proveer (...) y la mujer tiene que ocuparse de las labores de la casa". De esta manera, se buscaba desnaturalizar la visión tradicional que considera a las mujeres como principales responsables del trabajo doméstico y de la provisión de bienestar y cuidados. En este sentido, aunque no se interrogó la familiarización de la provisión de bienestar, sí se cuestionó que las mujeres nacieran "sabiendo cuidar niños y limpiar casas", en un intento de desencializar las funciones socialmente atribuidas a varones y mujeres en el seno de lo que podría entenderse como la familia nuclear moderna. En línea con esto, la docente presentó como deseable la corresponsabilidad de las tareas hacia el interior del hogar sosteniendo, por ejemplo, que "las personas tienen que lavar los platos más allá del género con el que te identifiques".

En consonancia con la visión de los/as adultos sobre estas publicidades algunos/as jóvenes sostuvieron, por ejemplo, que el "spot" B mostraba que "no sólo la mujer (...) sino también los hombres pueden ocuparse de las labores de la casa", que "el hombre también puede limpiar". En una intervención en este mismo sentido, la alumna Lourdes sostuvo respecto al anuncio $\mathrm{C}$ que:

"está buena la publicidad porque no te muestra que es una casa, algo rutinario, que estás ahí... y lo tiene que hacer un hombre o está la mujer haciéndolo. Sino más como un desafío, que no va a ser algo cotidiano..."

Este tipo de intervenciones fueron las de mayor duración y las que recibieron comentarios positivos por parte de los adultos. Sin embargo, en el intercambio en torno a estos spots, los adultos en ocasiones dieron por sabidas las interpretaciones que los/as estudiantes hacían de una publicidad, atribuyendo de antemano las posibles diferencias de perspectivas a una distancia generacional. En palabras de "la coordi":

viendo por ejemplo esta publicidad, personas más de nuestra edad, adultos (...) pensé necesariamente que faltaba una mujer ahí. Por ahí ustedes ya lo toman como no, no falta nada. No es que hace las cosas de una mujer, hace las cosas de un hombre y una hija (Leila, sobre spot B).

Question, Vol. 1, N. ${ }^{\circ}$ 63, julio-septiembre 2019. ISSN 1669-6581 
Analía Inés Meo, Lucía Elena Cavalo Relaciones de género en la escuela: entre la desnaturalización de los “micromachismos” y la reproducción de perspectivas binarias

Mientras en algunas intervenciones la visión de adultos y jóvenes sobre este tema parecieron confluir, en otras se distanciaron. Algunas opiniones de los/as estudiantes pusieron en evidencia que existen formas muy arraigadas de entender el cuidado y el trabajo doméstico como algo privativo de las mujeres. Estas formas se inscriben en discursos sociales que constituyen subjetividades y relaciones, y que se entraman en relaciones de poder históricamente constituidas (Hall, 2001). Este tipo de intervenciones de los/as alumnas/as dan cuenta de maneras hechas cuerpo de concebir las relaciones entre los géneros, que resisten incluso a la intención y/o esfuerzo consciente y activo de transformarlas:

Estudiante varón: "[El spot B] está mostrando a un hombre haciendo todas las labores de la casa.... de una mujer (agregó en voz baja)

Leila: ¿Todos los labores de...?"

Estudiante varón: que le corresponden a la mujer (contestó con timidez)

Leila: ¡¿Pero por qué metemos a la mujer acá?! (Expresó con tono de indignación)

Este comentario, que en principio estaría denotando desacuerdo, fue acallado sin llegar a ser interrogado. Este tipo de intervenciones se vinculan con experiencias y saberes situados que se anclan en contextos sociales e históricos específicos, y configuran matrices interpretativas, apreciativas y cognitivas que orientan prácticas, deseos e identidades, que sería valioso conocer.

\section{Control económico de los varones respecto de las mujeres}

Situaciones similares se produjeron tras la visualización del anuncio $D$, en el que los esfuerzos de adultos estuvieron destinados a sensibilizar a los/as estudiantes sobre la "violencia económica". De acuerdo con Segato (2010), el control económico constituye una de las formas más comunes de "violencia moral" contra las mujeres en América Latina. Para esta autora, este tipo de violencia forma parte de los mecanismos socialmente legitimados para garantizar la 
Analía Inés Meo, Lucía Elena Cavalo Relaciones de género en la escuela: entre la desnaturalización de los “micromachismos” y la reproducción de perspectivas binarias

perpetuación de las jerarquías de género. La misma consiste en el cercamiento y la coacción de la libertad económica de la mujer, volviéndola dependiente en esta esfera de otros/as.

\section{SPOT D}

La misma mujer del spot A se encuentra en un cambiador en un local de venta de ropa, probándose un vestido. Tras mirarse varias veces al espejo, llama por handy a un hombre -su pareja- a quien le comunica el precio del vestido. Él, desde su casa, con gesto serio y cuerpo erguido, ordena la compra del mismo enunciando la palabra "proceda" y le indica que lo pague en cuotas.

En línea con la definición propuesta por Segato, los/as adultos ofrecieron una lectura unívoca de esta publicidad: el varón de la pareja controlaba el dinero y con ello disponía y administraba del de su mujer. Por este motivo, según la "coordi" en este "spot" era él quién "le da autorización [para adquirir el vestido] y además le dice cómo lo tiene que comprar". En esta misma línea interpretativa, sostuvo que "lo que se repite siempre es que... la plata es del hombre, que la autorización la da hombre, que la esposa se da los gustos...".

Por otro lado, al analizar esta publicidad, Ricardo hizo referencia al tipo de lenguaje visual y verbal que utilizaban los protagonistas que él vinculaba a relaciones jerárquicas propias de una organización militar:

“(...) la dinámica que usaron para esta publicidad en realidad es una dinámica castrense. Es una dinámica militar. ¿Sí? Están hablando... por un aparato de comunicaciones, están hablando en términos castrenses. ¿Sí? castrense es...Militares... ¿ok? Entonces hay todo una ideología de un orden ¿sí? entonces la propaganda está diciendo mucho más de lo que dice".

Concluida su intervención, un alumno compartió una interpretación diferente de la misma situación:

Estudiante varón: "No, pero por ahí la tarjeta es del tipo"

Question, Vol. 1, N. ${ }^{\circ}$ 63, julio-septiembre 2019. ISSN 1669-6581

Instituto de Investigaciones en Comunicación | Facultad de Periodismo y Comunicación Social | Universidad Nacional de La Plata 
Analía Inés Meo, Lucía Elena Cavalo Relaciones de género en la escuela: entre la desnaturalización de los “micromachismos” y la reproducción de perspectivas binarias

Ricardo: “¿eh?”

Leila: ¡¿por qué es la tarjeta del tipo?! ¡¿Cómo sabes vos que es la tarjeta del tipo?!

Estudiante varón: "Pero no sé, por ahí está a nombre del hombre y..."

Ricardo: "Si está a nombre del hombre no la puede usar porque está a nombre del hombre"

Otros/as estudiantes también se manifestaron en desacuerdo con la lectura de quienes condujeron la actividad. Los primeros insistieron en que desde su perspectiva el llamado que la mujer del "spot" le hacía a su marido, en plena compra de un vestido, respondía a que "la plata es de los dos" y que, por lo tanto, era necesario "consultar" entre ellos para qué y cómo utilizarla. En cambio, la "profe" y el docente de técnica, de maneras diferentes, relativizaron el valor de estas miradas. A continuación, incluimos este diálogo:

Estudiante mujer: "Perdonáme ¿puedo hablar? Bueno, para mí la segunda no me parece para nada machista porque son una pareja y las parejas se tienen que consultar qué cosas tienen que comprar y qué cosas no...o sea consultar en qué van a gastar y asi".

Leila: "Está bien. Vos lo analizaste como que se estaban consultando. Yo lo interpreté como que el hombre le daba la autorización" (Interrumpe la frase porque un chico empieza a hablar en voz muy fuerte y a taparla).

Leila: "Pará. No lan. Estoy hablando yo. Eh... pero está bien si vos diste otra explicación y la explicación de que se consultan para vos es válida ¿Quien más pidió hablar a ver?”.

(Mientras ella decía esta frase lan siguió hablando al mismo tiempo que ella. Leila casi gritaba para que la oyeran).

José: “¿Juampi?”

Estudiante varón: "Ya está. Ya pasó lo que iba a decir."

José: ¿ ¿Vamos ¿Vemos la próxima...?" Ricardo: "Es cierto ¿está? pero eso es lo que le llama la lectura llana. Pero en realidad involucra todo el otro contexto, involucra justamente lo contrario." Leila: "Bueno, jvamos a ver otra!" 
A pesar de los intentos que durante esta actividad realizaron los/as adultos por trascender la perspectiva adulto-céntrica que ha dominado históricamente la organización de la escuela moderna, en estos intercambios se aprecian dificultades para dialogar, escuchar y/o habilitar las interpretaciones que hacen los/as estudiantes sobre las relaciones de género. Al respecto, podemos hipotetizar que la falta de tiempo, las características de la actividad y los atributos históricamente construidos del rol docente -como inculcador de saberes legítimos antes que exploradores/as de sentidos y disonancias- podrían estar interviniendo en estas reacciones involuntarias frente a la disonancia -las que tienen lugar durante interacciones rápidas, en donde las formas de responder son casi automáticas, sin mediaciones de la consciencia reflexiva-. Por ejemplo, al final del anterior extracto se evidencia que Ricardo califica de "lectura llana" a la mirada de los/as jóvenes, en el sentido de falta profundidad y que, a manera de velo ideológico, no es capaz de reconocer aquello que es verdadero y que es necesario develar (y sería accesible a los adultos). Los comentarios de los/as jóvenes, por su parte, apuntaron a desencializar las jerarquías entre varones y mujeres -ofreciendo así una lectura disonante, alternativa, a la propuesta por los adultos sobre lo que busca representar la publicidad-.

\section{Cuerpo femenino legítimo y mirada masculina}

La discusión que se produjo a partir de la visualización del spot E giró en torno a la violencia simbólica. Esta publicidad fue producida por un canal televisivo de deportes que publicitaba a "la liga femenina de básquet". Consiste en una sucesión de distintas tomas de una mujer joven en ropa deportiva ajustada al cuerpo, realizando diversos movimientos de básquet (por ejemplo, tirando al cesto).

Luego de la visualización de este "spot", la docente invitó a los/as estudiantes a interrogar la forma en que las mujeres aparecían representadas en el mundo de la publicidad, haciendo hincapié en este caso en los estereotipos en torno a la belleza y al cuerpo femenino. A continuación, incluimos una cita extensa que presenta los diálogos que se sucedieron:

Estudiante varón: "¿Están todas así de buenas o esa...? "

José: "A ver, ¡ahí está! ¡Ahí está! ¡Esperen, esperen! hay una cuestión puntual que dice Juan. La pregunta que dice Juan dice "¿están todas así de buenas?", yo no voy a traducir, “¿o no? ¿Qué quiere decir? que en esa propaganda lo que está mostrando y lo que quiere vender, es 
Analía Inés Meo, Lucía Elena Cavalo Relaciones de género en la escuela: entre la desnaturalización de los “micromachismos” y la reproducción de perspectivas binarias

que las chicas tienen que tener ciertos estándares físicos para poder competir en la liga femenina de básquet".

Estudiante varón: "tiene que ser alta..."

Estudiante mujer 1: “¿Pero en algún momento te dijo que tenés que ser así?"

Ricardo: "No, no, no. A ver, pero el tema es ¿están promocionando la liga de básquet? o sea ¿yo veo un equipo jugando? ¿o veo una mu..., como dicen una, ya desde el vamos, una mina. Una mina que está re buena. Y lo dice otra mujer"

Estudiante varón: "Seee"

Ricardo: "¿sí? entonces a quién está dirigido a esa propaganda? ¿Al público en general o a nosotros los babosos?"

Estudiantes contestan al unísono: "a los babosos, a los babosos"

Como se muestra en este diálogo, una sola estudiante problematizó la relación directa entre imagen publicitaria y sus efectos respecto a la generización femenina (“¿pero en algún momento te dijo que tenés que ser así?"). Algo que no pudo ser retomado por los/as adultos. El resto de los comentarios fueron hechos por estudiantes varones los que, en distintos momentos, parecían encarnar y sostener la lectura que José y Ricardo hicieron de la publicidad. Según ellos, la publicidad buscaba producir subjetividades femeninas en el deporte que actualizaban discursos sociales sobre el cuerpo femenino (expresados por el discurso visual que mostraba a una mujer alta, blanca, de figura torneada y flaca). Los adultos invitaron entusiastas a explayarse a aquellos estudiantes que expresaron dichos que ratificaban sus propias lecturas:

"José: “¡Esperen, esperen! A ver... ¡Juampi! Él va a dar su opinión sobre esta perspectiva que es lo que vio en la propaganda."

Estudiante varón (Pedro): "para mí, ya como dijo Leila. Está dirigido al público masculino y... escondido muy escondido es como si... el hombre tendría que enamorarse de esa mujer"

En estos diálogos es posible ver cómo la interpretación sobre la publicidad buscaba cuestionar la producción de la mujer como objeto de apreciación para la mirada masculina y a los varones como "babosos". Estas maneras de referirse a la publicidad contribuyen (de maneras no buscadas) a excluir del campo de sentido posible a otros tipos de masculinidades y de sexualidades. En este caso, estudiantes varones expresaron visiones convergentes con las de los adultos de su mismo género. Estos jóvenes y adultos fueron hablados por un discurso social que contribuye a concebir y performar a los varones como un grupo homogéneo y 
Analía Inés Meo, Lucía Elena Cavalo Relaciones de género en la escuela: entre la desnaturalización de los “micromachismos” y la reproducción de perspectivas binarias

heterosexual. Asimismo, este discurso se imbrica con otro sobre la belleza femenina que define, clasifica y jerarquiza a las mujeres según sus cuerpos y hexis corporales, a partir de la distancia o proximidad de sus atributos físicos respecto de las representaciones dominantes de la "belleza femenina" que atraviesan diferentes campos sociales y culturales.

Durante la conversación sobre este spot, la gran mayoría de las chicas no participó. Como vimos, sólo una cuestionó el poder de las publicidades sobre las maneras en que las jóvenes ven su cuerpo (suponiendo así agencia o capacidad crítica por parte de las mujeres). Podría pensarse que la forma de poner en escena a la mirada masculina sobre el cuerpo de las mujeres antes que propiciar la toma de la palabra por parte de las estudiantes, las llamó a silencio y, en este sentido, no las habilitó para compartir experiencias y tensiones respecto de las formas dominantes de definir la belleza femenina y de posicionarse frente a ella (algo que sería, por cierto, una compleja tarea de llevar adelante en el poco tiempo en el que se realizó esta actividad).

\section{La generización de las manifestaciones emocionales en debate}

La visualización del anuncio $\mathrm{F}$ contribuyó a visibilizar discursos sociales que buscan producir a las mujeres como "lloronas", como débiles e incapaces de controlar sus emociones e, implícitamente, a los varones como sujetos fuertes, en control de sus emociones que no necesitan expresarlas a través del llanto. A continuación, reproducimos el intercambio central en torno a esta publicidad:

José: "A ver, ¿Qué se vio en la propaganda?"

Estudiante varón (José): "Dice que las mujeres son las únicas que lloran pero también los varones lloramos".

Ricardo: "ioh! que tierno"

Estudiantes: "iAaah!" (Dijeron en tono tierno, como si se estuvieran burlando de lo dicho por José. Luego ríen).

Marga: “¡Acá hubo un momento de micromachismo! Estuvo buenísimo porque vos desarmaste la situación y el profesor de técnica volvió a caer en la misma. ¿Por qué?"

Ricardo: "Obvio"

Marga: "Porque volvió a decir "que tierno". Y esto es un momento también de micromachismo" Estudiante varón (Juan): “¡Machista! ¡Andate!” 
Analía Inés Meo, Lucía Elena Cavalo Relaciones de género en la escuela: entre la desnaturalización de los “micromachismos” y la reproducción de perspectivas binarias

Ricardo: "Obvio. Ahí está. Bueno".

$(\ldots)$

Estudiante varón: “¿Qué es micromachismo?”.

"Coordi" varón: "A ver. Juan. Juan dice que... no solo las mujeres pueden llorar, sino que los hombres también. ¿Qué piensa al respecto el resto del grupo? a ver..."

Estudiante mujer (Lourdes): "Tiene razón la publicidad porque las mujeres somos mucho más... sentimentales".

Estudiante mujer: "pero es algo cultural"

Leila: "¿Los varones no tienen sentimientos?"

Estudiantes: “isi!"

Leila: "No, pero entonces por ahí, entonces lo que pienso, si los chicos dicen que tienen sentimientos no es que somos más sentimentales las mujeres. Es más permitido para las mujeres mostrar lo que sentimos"

(...)

Estudiante mujer (Lourdes): "Que lloramos por todo".

Ricardo: (...)“... yo lo hice a propósito cuando dije "qué tierno”. ¿Sí? qué es lo que decía Leila. ¿Qué estoy diciendo? ¿Qué me da a entender eso? ¿Por qué poné así?"

Estudiante mujer (Lourdes): "las mujeres lloran más"

Ricardo: “¿pero eso es así?"

Estudiante mujer (Lourdes): "Si"

Estudiante mujer: "No, porque un varón puede llorar tanto como una mujer", dijo en voz muy baja.

En este intercambio puede observarse tensiones entre posiciones discursivas contrastantes. La selección de la publicidad buscaba ilustrar que la sociedad regula las emociones de los varones de maneras diferentes a las de las mujeres $y$, que por este motivo, los primeros no pueden llorar (porque no se les permite que lo hagan aunque tienen similares sentimientos a los de las mujeres). Las discusiones muestran que este tipo de discursos es subjetivante para algunas mujeres ("las mujeres lloran más", "somos más sentimentales"); mientras que no lo es para algunos varones ("los varones también lloramos") y otras estudiantes ("un varón puede llorar tanto como una mujer"). Asimismo, en este intercambio, la expresión de las emociones a través del llanto fue feminizada y desvalorizada por el docente de técnica y varios estudiantes varones. El docente, con tono burlón, calificó de "tierno" al estudiante que expresó que los "varones también lloramos"; reacción que fue secundada por un grupo de varones. Al mismo tiempo, la reacción de Marga, la asesora pedagógica, estaría dando cuenta de contradicciones 
Analía Inés Meo, Lucía Elena Cavalo Relaciones de género en la escuela: entre la desnaturalización de los “micromachismos” y la reproducción de perspectivas binarias

que se despliegan en el devenir de este diálogo: según ella, la intervención del docente varón contribuía a escenificar una jerarquía entre varones y mujeres que la "actividad" buscaba cuestionar, al mismo que reificaba estereotipos tradicionales de género.

Como mencionamos, varias jóvenes entendieron que la publicidad "tiene razón", y que las mujeres lloran más que los varones. Estas intervenciones pueden ser consideradas como huellas de discursos sociales que organizan las relaciones de género con fuerte poder performativo en la constitución de subjetividades. Huellas que una de las estudiantes entiende como resultado de la cultura, antes que de la naturaleza. Así, este extracto evidencia que, a pesar de los esfuerzos conscientes de los/as adultos por cuestionar y desnaturalizar las relaciones tradicionales entre los géneros, jóvenes y adultos encarnan (de maneras contradictorias e inconscientes) discursos sociales dominantes y subalternos acerca de cómo deben pensar, sentir y actuar los varones y las mujeres. Discursos que es difícil deconstruir y trastocar ya que se encuentra hecho cuerpo, moldeando maneras de pensar, actuar y sentir.

\section{Violencia contra las mujeres}

Luego de la visualización y discusión de las "spots", los/as jóvenes fueron invitados/as a escribir en unas cartulinas "un mensaje o algo que nosotros queramos", en base a los temas conversados. Además, comentó que estas producciones iban colocarse en la puerta del colegio con el fin de "(...) enseñarle a los demás. Más que nada a los adultos que son los que por ahí (...) tenemos la cabeza más cerrada en este sentido". En un clima más distendido, los/as alumnos/as se agruparon en pequeños grupos y comenzaron a conversar entre sí y a elaborar carteles. El y la profesor/a coordinador/a se desplazaron por el aula, manteniendo conversaciones informales con los/as estudiantes mientras los observaban trabajar.

Durante esta tarea algunos/as estudiantes referenciaron situaciones de violencia en las que, poniendo a las mujeres como sujetos del ejercicio de la violencia, tensionaban la visión promovida por los/as adultos/as sobre ésta. En este sentido, se dio un diálogo entre una alumna que sostuvo en forma insistente ante la docente y un pequeño grupo de compañeros y compañeras la siguiente visión sobre el ejercicio de la violencia:

Estudiante mujer 1: pero así como mujeres agreden a... así como hay hombres que agreden a las mujeres también hay mujeres que a los hombres. 
Analía Inés Meo, Lucía Elena Cavalo Relaciones de género en la escuela: entre la desnaturalización de los “micromachismos” y la reproducción de perspectivas binarias

Leila: sí. Lo que pasa es una cuestión de que los hombres no son una minoría. Te explico esto. Hay minorías sociales, por ejemplo... siempre hablamos del tema de... el holocausto. Los judíos....

Estudiante mujer 1: ¿de qué?

Leila: El holocausto. Los judíos son una minoría en el sentido que son, una parte de la sociedad oprimida, asesinada de hecho, que donde se le vulnero los derechos durante mucho tiempo. Y todavía hoy hay un montón de lugares y de situaciones en las que se le vulneran los derechos. Son discriminados, son tratados como diferentes... las mujeres, históricamente, se construye un rol de mujer como minoría, es decir que sus derechos están vulnerados. Y los hombres no son una minoría. El hombre es el que ejerce el poder y el dominio sobre parte de la sociedad, incluso hombres. Sobre incluso hombres pero también sobre la mujer. Entonces, la agresión sobre un hombre puede ser, pero es un caso particular, que no tiene que ver con la condición de que es hombre. La agresión hacia la mujer cobra otra dimensión porque se la ataca por solo el hecho de ser mujer. ¿Está bien? Entonces no es que... no sé, vos le podes pegar a alguien porque te enojaste, porque te hizo algo malo... y alguien y te puede pegar a vos por eso o por solo el hecho de ser mujer, porque en la sociedad ser mujer implica determinadas cosas que a veces no... no se... no se respeta que tengan libertad, que tenga...

De este modo la docente presentó a la "violencia de género" como una relación que necesariamente involucra dos términos dicotómicos, es decir, a varones y a mujeres. Como un fenómeno unidireccional, en el cual los varones son los perpetradores de una violencia que tiene por objeto a las mujeres -víctimas efectivas o potenciales, objetos pasibles de ser violentadas). Esta representación (producida por discursos sociales contemporáneos dominantes en torno a la "violencia de género") desdibuja la capacidad de agencia de las mujeres tanto como sujetos que pueden interrumpir el ejercicio de la violencia, como personas capaces de ejercer la violencia sobre otros/as. Luego del esfuerzo realizado por la docente por intentar re-encauzar la visión de la estudiante, la misma joven mencionó -sin obtener respuesta- otra situación de violencia que no tenía como a los varones como protagonistas, al afirmar que "también la misma mujer agrede a otra compañera mujer". Asimismo, en el marco de una conversación que siguió a la antes expuesta dos estudiantes manifestaron que: 
Analía Inés Meo, Lucía Elena Cavalo Relaciones de género en la escuela: entre la desnaturalización de los “micromachismos” y la reproducción de perspectivas binarias

Estudiante mujer: Acá las mujeres más que nada... controlan a los varones

Estudiante varón: Sí, nos golpean.... Acá debería ser "Ni Uno Menos"

Más allá del tono jocoso de estas intervenciones, es interesante que los/as chicos/as hayan cuestionado el carácter estático y unidireccional de las relaciones de dominación así como del ejercicio de la violencia física y simbólica. Este tipo de interrupciones, que cuestionan las visiones presentadas por los adultos -visiones fundadas en discursos que nos constituyen, más allá de nuestra intencionalidad y consciencia-, nos ofrecen invitaciones a conocer las perspectivas de los/as jóvenes distintas de las presentadas como verdaderas (tal como la sustancialización de la violencia en los cuerpos varoniles).

\section{A manera de cierre}

El análisis de esta "actividad”, ofrece algunas claves para comenzar a indagar los esfuerzos que una escuela está realizando para problematizar las relaciones de género de sus estudiantes, a partir de prescribir visiones acerca de lo deseable y lo apropiado en las relaciones entre varones y mujeres. Desde nuestra mirada, la "actividad" constituye una huella del modo en que se estaría produciendo una representación de las relaciones deseables (y no) entre varones y mujeres en la escuela. En otras palabras, una generización de prácticas y discursos.

En el transcurso de la actividad, por un lado, los/as adultos encarnaron discursos que habilitaron y promovieron en los/as jóvenes cuestionamientos de visiones jerárquicas y esencialistas sobre las relaciones de género, que se pretendía que éstos compartieran y reprodujeran. Por el otro, pareciera que los adultos son hablados por discursos sociales que tienden a silenciar o a marginalizar las visiones y/o inquietudes de los/as estudiantes que introducen puntos de fuga, tensiones y contradicciones que no es posible integrar. De manera no buscada, este tipo de encuentros podría estar dificultando el reconocimiento de las maneras en que los/as jóvenes significan las relaciones de género y las formas en las que interpretan las jerarquías entre hombres y mujeres así como entre mujeres y entre varones. Asimismo, esta resistencia a integrar aquello que irrumpe y no encaja en las formas "apropiadas" de entender las relaciones de género por parte de los/as adultos podría estar apuntado a límites en las intervenciones pedagógicas puntuales que buscan alterar códigos "machistas", y con ello 
Analía Inés Meo, Lucía Elena Cavalo Relaciones de género en la escuela: entre la desnaturalización de los “micromachismos” y la reproducción de perspectivas binarias

propiciar la apropiación de maneras "no machistas" de relacionarse entre varones y mujeres. En ocasiones, algunos adultos asumen que comprenden lo que los/as estudiantes están expresando antes de indagar sobre incomodidades y timideces. En este sentido, no fue posible interrogar aquello que se está diciendo o mostrando con el tono de voz y con el cuerpo (tanto de estudiantes como de adultos). Esta aparente dificultad puede estar vinculada a variedad de cuestiones (todas ajenas a las voluntades e intencionalidades de los actores que con compromiso llevan adelante estas actividades). Entre ellas, y a manera de clave interpretativa identificamos, por un lado, la falta de tiempo para trabajar estos temas (antes, durante y después de los encuentros); la cantidad de estudiantes que comparten esta actividad; y la falta de espacios institucionales para problematizar los supuestos que los/as adultos tienen sobre las relaciones de género y generacionales. Por el otro, las interacciones rápidas, los decires y gestos que acontecieron durante esta actividad se nutrieron de discursos sociales antagónicos en torno a las relaciones de género. Discursos sociales que nos constituyen como sujetos y hablan a través de nosotros/as. En particular, hipotetizamos que la operación de la lógica pedagógica escolar contribuyó a que quienes llevaron adelante esta actividad asumieran un rol de inculcar saberes que fueron tomados y presentados como verdaderos, antes que resultantes de una co-producción entre adultos y jóvenes. Este camino supondría, por un lado, la visibilización y cuestionamientos de las propias perspectivas y de las ajenas -nuestro "machismo", nuestras propias condiciones de vida que promueve ciertas miradas, y las dificultades que tenemos en desandar formas que tienden a reproducir miradas binarias sobre las relaciones de género, etc.-. Por el otro, este derrotero incorporaría el reconocimiento de nuestras miradas y sentires como productos socio-históricos que se encarnan en nuestros cuerpos (más allá de nuestra consciencia y control). Las maneras en las que se sortean estos obstáculos son colectivas y trascienden el ámbito escolar. Este primer análisis se propone abonar de manera exploratoria acercando preguntas e interrogantes, antes que claras respuestas y cursos de acción para seguir. Éstos se forjan con otros (jóvenes y adultos) en y fuera de la escuela.

Notas

Las autoras agradecen a autoridades, docentes, estudiantes y personal no docente de la escuela por su permanente generosidad y colaboración. En particular agradecemos las lecturas críticas de dos profesores coordinadores que nutrieron este análisis. Las omisiones u errores son nuestras. 
Analía Inés Meo, Lucía Elena Cavalo Relaciones de género en la escuela: entre la desnaturalización de los “micromachismos” y la reproducción de perspectivas binarias

(1) El entrecomillado es utilizado para referirse a expresiones utilizadas por distintas personas durante el trabajo de campo.

(2) Esta investigación de carácter etnográfico se inició en 2015 e implicó visitas semanales al colegio por parte de distintos investigadores del equipo. Para el análisis de la "actividad", se llevó a cabo un análisis temático a partir de la lectura pormenorizada del registro de observación. Agradecemos muy especialmente a autoridades, docentes (incluyendo a los "profesores coordinadores"), estudiantes, y en especial a la "profe" a cargo de la actividad analizada. La generosidad y paciencia de todos/as ellos/as han sido aspectos centrales de nuestro trabajo.

(3) Para garantizar la confidencialidad y el anonimato de los/as integrantes de la escuela, en esta publicación sus nombres fueron reemplazados por pseudónimos.

\section{Bibliografía}

Alonso, G.; Herczeg, G. y Zurbriggen, R. (2009). Cuerpos y sexualidades en la escuela. Interpretaciones desde la disidencia. En Villa, A. (comp.). Sexualidad, relaciones de género y de generación. Perspectivas histórico-culturales en educación. Buenos Aires: Noveduc Libros.

Goldstein, B. y Glejzer, C. (2008). Las preguntas de los y las adolescentes... comenzar por escuchar. En Morgade G. y Alonso, G. (comps.). Cuerpos y sexualidades en la escuela. De la "normalidad" a la disidencia. Buenos Aires: Paidós.

Hall, S. (2001). Foucault: Power, knowledge and discourse. En Wetherell, M.; Taylor, S. y Yates, J. (eds.). Discourse theory and practice. A reader. Londres: Sheffield Hallam University.

Morgade, G. (2002). Aprender a ser mujer, aprender a ser varón: relaciones de género y educación: esbozo de un programa de acción. Buenos Aires: Noveduc Libros.

Ramos, G. (2009). Discurso/s que construyen sexualidades en las escuelas medias de la Ciudad de Buenos Aires. En Villa, A. (comp.). Sexualidad, relaciones de género y de generación. Perspectivas histórico-culturales en educación. Buenos Aires: Noveduc Libros.

Segato, R. L. (2010). Las estructuras elementales de la violencia. Ensayos sobre género entre la antropología, el psicoanálisis y los derechos humanos. Buenos Aires: Prometeo Libros. 
Ramos, G. y Román, C. (2008). La "prevención” como cuestión de las mujeres adolescentes. En Morgade G. y Alonso, G. (comps.). Cuerpos y sexualidades en la escuela. De la "normalidad" a la disidencia. Buenos Aires: Paidós.

Wainerman, C. y Chaml, N. (2014). Sexualidad y escuela. Perspectivas programáticas posibles. Archivos Analíticos de Políticas Educativas, 22(45). Recuperado de https://epaa.asu.edu/ojs/article/view/1744/1263 